\title{
Avaliação da colaboração logística entre uma distribuidora e seus fornecedores
}

J oséGeraldo Vidal Vieira (UFV) - jose-vidal@ufscar.br

DaniellePiresCoutinho (UFV) - danipcoutinho@gmail.com

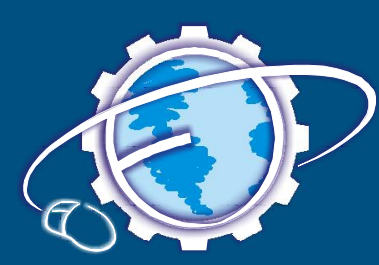

Resumo

Neste trabalho foram propostos indicadores de colaboração logística que servem como parâmetro para avaliar os fornecedores de uma distribuidora e entender a relação da parceria. A partir da revisão de literatura e de um estudo de caso em uma distribuidora, foram definidos quatro grupos de elementos que serviram de base para uma pesquisa quantitativa. D ois grupos foram analisados, estatisticamente, por meio de medidas centrais e dois pela análise fatorial. 0 s resultados indicaram que os elementos de integração interpessoal (G rupo 2) e aquel es referentes a pedido (Grupo 4) foram os que apresentaram maior média e pouca variabilidade dos dados. Para melhor entendimento dos elementos operacionais de desempenho logístico ( $G$ rupo 1) e dos elementos referentes a informações logísticas e comerciais (G rupo 3) foram criados indicadores, a partir das cargas fatoriais, que revelaram subgrupos com maior ou menor grau de interferência na colaboração logística. D o ponto de vista dos fornecedores, embora se tenha uma satisfação quanto ao relacionamento interpessoal, o indicador de reuniões logísticas apontou que maior contato por meio de reuniões pode mel horar a troca de informações logísticas e comerciais e, possivelmente, contribuir para uma melhora do desempenho logístico do fornecedor.

Palavras-chave: Colaboração, el ementos de desempenho logístico e análise fatorial.

\section{ASSESSMENT OF COOPERATION BETWEEN THE LOGISTICS AND ITS DISTRIBUTOR'S SUPPLIERS}

\section{Abstract}

This paper suggests collaboration logistics indicators that it can be used as a parameter to evaluate the suppliers of a distributor and understand the partnership relationship. From the literature review and a case study in a distributor, were defined four groups of elements. From case study was developed a quantitative research. Two groups were statistically analyzed through central measures and two groups through factorial analysis. The results indicated that the elements of interpersonal integration (G roup 2) and those relating to manager order (G roup 4) showed higher average and low variability of the data. F or better understanding of the operational elements of performance logistical (Group 1) and those relating to logistics and trade information (G roup 3) were created indicators, from loads factorial, which revealed subgroups with greater or lesser degree of interference in collaboration logistics. F rom view of suppliers, although it has a satisfaction about the interpersonal relationships, the indicator of meetings logistics showed that more contact through meetings can improve the exchange of information and trade logistics, and possibly help an improvement of the performance of the logistics provider. Keywords: Collaboration, logistics performance elements and factorial analysis. 


\section{INTRO DUÇÃO}

No Brasil, as últimas décadas foram marcadas por mudanças no ambiente empresarial, ocasionadas principalmente pela abertura do mercado. Alguns aspectos desse processo foram particularmente relevantes para o setor varejista e seus fornecedores, tendo em vista a crescente entrada de produtos importados, o aumento dos investimentos externos e a maior concentração do mercado por meio de processos de fusão e aquisição.

Compondo o ambiente competitivo em que se encontram as empresas brasileiras, os aspectos econômicos, principalmente a questão dos altos custos de capital no país, vêm exercendo forte pressão para o investimento em logística ou de parcerias entre os participantes da rede de uma cadeia de suprimento. Como consequiência, melhorar o sistema logístico passou a ser fator imperativo para ganho de competitividade das indústrias, distribuidores e varejistas. Isso tem feito com que surja uma série de iniciativas para aumento da eficiência operacional, além de uma quantidade cada vez maior de projetos específicos de resposta rápida envolvendo indústria e varejo, bem como de replanejamento de redes de distribuição.

Como a competição não acontece somente entre uma empresa e outra, mas também entre cadeias de suprimentos, a avaliação do desempenho logístico assume papel relevante na avaliação da cadeia. Um excelente desempenho, nos níveis de serviço oferecido ao cliente, tende a aumentar, de modo substancial, o valor agregado em toda a cadeia de suprimentos. Portanto, é necessário conduzir pesquisas com o objetivo de investigar como as empresas estão avaliando o nível de serviço logístico prestado pelos seus fornecedores, assim como pesquisar os indicadores mais apropriados para avaliar o desempenho logístico das empresas.

Nesse novo contex to, a eficiência logística, por meio da colaboração, ganha cada vez mais importância. Enquanto pelo lado do varejo se verifica a tendência de aumento nos níveis de exigência no que diz respeito à entrega, pelo lado da indústria ocorre a busca pela adaptação dos sistemas de distribuição aos parâmetros de mercado, levando às variações nos indicadores de nível de serviço logístico entre indústria e varejo. Isso faz que haja intensa troca de informações, ações conjuntas e forte relacionamento interpessoal entre os parceiros, para que se tenha melhora significativa das operações logísticas.

Este estudo, inspirado nos trabalhos de Zanquetto et al. (2003), Simatupang e Sridharam (2005) e Vieira et al (2007), buscou avaliar os fornecedores de uma distri- buidora, usando variáveis que complementam a pesquisa de Vieira (2006), ou seja, variáveis peculiares à realidade dessa empresa.

O objetivo geral é avaliar o desempenho logístico dos fornecedores daquela distribuidora. Mais especificamente, pretendeu-se estabelecer indicadores de colaboração logística que sirvam de parâmetros para avaliar o desempenho logístico dos parceiros da distribuidora e conhecer o relacionamento dela e de seus parceiros.

O desempenho logístico dos fornecedores da distribuidora se mostrou muito heterogêneo; alguns atendiam a todos os fatores, como: entregas completas, entregas sem erros, disponibilidade de produtos, cumprimento da agenda de entrega e cobertura de entrega. No entanto, existem os que deixam a desejar em alguns desses fatores, comprometendo a imagem da empresa diante de seus clientes. Podem-se destacar situações em que um produto de alto giro chegou a um nível de estoque nulo por falta de possibilidade de ressuprimento do estoque. É importante destacar que, mais do que comparar e monitorar o desempenho logístico entre os parceiros, os indicadores de desempenho servem para aproximar os participantes da cadeia de uma cadeia de suprimento em torno de um diálogo, o que permite maior contato entre as áreas funcionais das empresas, tanto no campo operacional (comunicação diária) quanto tático (desenvolvimento de projetos e conhecimento das dificuldades e estratégias entre parceiros). Conseqüentemente, esse maior envolvimento se traduz em maior compartilhamento de informações e ações conjuntas, completando o ciclo de colaboração logística (VIEIRA, 2006).

Nesse sentido, este trabalho é importante, por pesquisar e avaliar os diferentes atributos de colaboração logística presentes no elo fornecedor-distribuidora, bem como certificar estudos já realizados nessa área, como descrito anteriormente.

\section{REVISÃO DE LITERATURA 2.1. Colaboração Logística}

Uma organização deve oferecer produtos e serviços aos clientes de acordo com as suas necessidades e exigências do modo mais eficiente possível. Dessa maneira, Ballou (2001) considerou que a missão da logística é "dispor a mercadoria ou o serviço certo, no lugar certo, no tempo certo e nas condições desejadas, ao mesmo tempo que fornece a maior contribuição à empresa".

Fazem parte do sistema logístico os seguintes componentes: serviço ao cliente, controle de estoque, 
processamento de pedidos, compras, tráfego e transporte, armazenagem e estocagem, entre outros (MENTZER; KONRAD, 1991; BALLOU, 2001; BOWERSOX, 2001).

Segundo Ballou (2001), alguns desses componentes fazem parte das chamadas atividades-chave (padrão de serviço ao cliente, transportes, administração de estoques, fluxo de informações e processamento de pedidos). Para Daugherty et al. (1996), as atividadeschave da logística, quando integradas, têm o objetivo de reduzir os custos com a distribuição e manter um bom nível de serviços ao cliente, ou seja, têm o propósito de fornecer valor para clientes e fornecedores. Esse valor é traduzido em tempo e lugar. Logo, o transporte adiciona valor de lugar aos produtos e serviços, enquanto estoque adiciona valor de tempo. Por exemplo, tem-se o transporte de produtos de mercearia de uma indústria para um centro de distribuição. Assim, a logística adiciona valor de lugar se esses produtos forem transportados seguramente em caminhões paletizados que reduzem perdas com o transporte (como produtos amassados e defeituosos, entre outros); adiciona valor de tempo quando esses produtos, bem acomodados na área de estocagem, podem ser consumidos durante todo o período de validade.

Novaes (2001) foi um pouco além, dizendo que a logística não agrega apenas valores de tempo e lugar, mas também de qualidade - o produto deve estar nas proporções desejadas dos consumidores (quantidade, forma e tamanho) e valor de informação (como formas de rastreamento de produtos, sistema de códigos de barra etc.). No entanto, para que tais atividades se realizem de forma eficiente, deve haver uma troca constante de informações entre os parceiros, relacionamento de confiança, ações conjuntas, enfim, relacionamento mais estreito (cooperativo ou de colaboração), para que o que foi delineado no campo estratégico seja executado pelas partes a um menor custo ou com menor esforço operacional.

Vieira (2006) ressaltou que os aspectos comportamentais de colaboração como confiança, interdependência, reciprocidade, flexibilidade e comprometimento são fundamentais para a realização de qualquer transação. Portanto, a colaboração - quando duas ou mais empresas trabalham juntas ao longo do tempo (com base na confiança, flexibilidade, reciprocidade, comunicação aberta, interdependência, comprometimento, conhecimento do parceiro e no longo tempo), por meio de decisões conjuntas, compartilhando informações logísticas e comerciais, custos e benefícios e com o objetivo de atender às necessidades dos clientes - é uma importante ferramenta estratégica para aumentar a sinergia entre os parceiros, com o intuito de reduzir custos logísticos e aumentar a competitividade das empresas (VIEIRA, 2006). Para o melhor entendimento sobre colaboração, destacam-se as pesquisas de: Anderson e Narus (1990), Heide e John (1992), Morgan e Hunt (1994), Ganesan (1994), Mohr e Spekman (1994) e Kumar (1996), entre outros.

Rey (1999) considerou que a logística é uma das atividades mais importantes como diferencial competitivo e o ponto mais crítico na formação de custos. Isso se reflete na ligação forte entre a política de serviços ao cliente e as políticas de estoque, transporte, material, distribuição e armazenagem e que só podem ser explicadas por meio de indicadores de desempenho. Essa autora também afirmou que, para medir o desempenho da função logística, é necessário ter, como marco de referência, os indicadores genéricos que a empresa utiliza como um todo. Esses indicadores têm sido construídos com base nos elementos que contribuem para melhorar a posição competitiva da empresa.

Entende-se que esses indicadores genéricos sejam formados por elementos estratégicos, táticos e operacionais, ou seja, indicadores que possam avaliar não só o desempenho operacional, mas também o relacionamento ao longo de uma parceria.

Vieira (2006) adotou o termo "colaboração logística" como o relacionamento fechado entre fornecedores e seus clientes varejistas (como consumidores diretos), de forma que esses fornecedores utilizem parâmetros para avaliar a distribuição de seus produtos e melhor atender as necessidades dos seus clientes varejistas. Tais parâmetros se referem aos elementos de colaboração no nível estratégico tático e interpessoal. A colaboração estratégica se refere às decisões das empresas na definição dos objetivos e compartilhamento de informações estratégicas (estoque, conhecimento das dificuldades e estratégias logísticas do parceiro, histórico de relacionamento e envolvimento da alta gerência na definição dos acordos logísticos, entre outros). A colaboração no nível tático se refere às ações realizadas em conjunto com o parceiro, às trocas de dados operacionais e às trocas de informações importantes para a melhora do desempenho logístico e redução dos custos logísticos. A colaboração no nível interpessoal se refere ao relacionamento interpessoal entre os agentes, com o objetivo de, cada vez mais, ampliar as áreas de contato e estabelecer a confiança. Esses elementos, quando agregados, formam o conceito de colaboração logística.

Vale ressaltar que, embora esse termo tenha sido utilizado recentemente, sobretudo na literatura brasileira, outros termos relacionados à logística colaborativa são 
descritos por diversos autores desde a década de 1980, como: Ohmae (1989), Ellram (1990), Spekman et al. (1998), Mentzer et al. (2000), Barratt e Oliveira (2001) e Bowersox et al. (2003), entre outros.

\subsection{Indicadores de desem- penho logístico}

A Fundação para o Prêmio Nacional da Qualidade define desempenho como:

Os resultados obtidos de processos e produtos que permitem avaliá-los e compará-los em relação às metas, aos padrões, aos referenciais pertinentes e a outros processos e produtos, mas comumente, os resultados expressam satisfação, insatisfação, eficiência e eficácia e podem ser termos financeiros ou não (FPNQ, 1995, p. 54).

Gerenciar é controlar. Sem controle não há gerenciamento. Sem medição não há controle (JURAN, 1992).

Indicador de desempenho é uma relação matemática que mede, numericamente, atributos de um processo ou de seus resultados, com o objetivo de comparar esta medida com metas numéricas pré-estabelecidas (FPNQ, 1995, p. 5).

Para melhorar os processos logísticos, deve-se compreender o que são medidas de desempenho, para que servem e como podem ser usadas como objetivo de controle e melhoria.

Para Hronec (1997), as medidas de desempenho são sinais vitais da organização, qualificando e quantificando o modo como as atividades ou outputs de um processo atingem suas metas. Assim, as medidas de desempenho respondem à pergunta: como você sabe?

Um sistema de indicadores de desempenho é definido como o conjunto de pessoas, processos, métodos e ferramentas que, conjuntamente, geram, analisam, expõem, descrevem, avaliam e revisam dados e informações sobre as múltiplas dimensões do desempenho nos níveis individual, grupal, operacional e geral da organização, em seus diversos elementos constituintes (MacedoSOARES; RATTON, 1999).

Baseado nesses sistemas de indicadores, existem características de desempenho de sistema logístico que servem como direcionadores de eficiência logística. Foram determinados alguns exemplos de características importantes da logística e medidas de desempenho correspondentes, de acordo com o que Moller e Johansen (1993) apud Bastos (2003) analisaram, como: disponibilidade, nível de desempenho, confiabilidade, flexibilidade, robustez, qualidade, produtividade e serviço. As empresas estão cada vez se conscientizando de que não é possível atender às exigências de serviço dos clientes e, simultaneamente, cumprir com os objetivos de custo da empresa sem trabalhar de forma coordenada, com outros participantes da cadeia de suprimentos (FLEURY; LAVALLE, 2000 apud ÂNGELO, 2005). Assim, os indicadores de desempenho logístico podem monitorar a qualidade das atividades logísticas internas à empresa ou a de seus parceiros (fornecedores). Quanto ao âmbito, podem ser:

(i) Interno: monitoram o desempenho dos processos internos à empresa. Ex.: giro de estoque, ruptura de estoque, pedidos perfeitos, tempo de ciclo do pedido, estoque disponível para venda, acuracidade de estoque, utilização das capacidades de estoque, pedidos por hora, custo por pedido, custos de movimentação e armazenagem, tempo médio de permanência do veículo de transporte, utilização dos equipamentos de movimentação, coletas no prazo e utilização da capacidade de carga de caminhões (ÂNGELO, 2005).

(ii) Externo: monitoram o desempenho dos serviços prestados pelos parceiros (fornecedores) da empresa. Ex.: entregas realizadas dentro do prazo, tempo de ressuprimento do fornecedor, entregas devolvidas parcial ou integrais, recebimento de produtos dentro das especificações de qualidade e atendimento do pedido realizado (ÂNGELO, 2005).

A necessidade de aprimoramento das relações entre empresas de uma cadeia fez surgir a preocupação de monitoramento de indicadores de âmbito externo. Muito mais do que ferramentas de acompanhamento do serviço prestado pelos parceiros da cadeia de suprimentos para possível negociação, os indicadores de desempenho logístico externo são fundamentais para a definição de políticas e processos internos que dependem do desempenho de seus parceiros. Além disso, eles são essenciais na coordenação de políticas que garantam a competitividade da cadeia de suprimentos.

Gunasekaram et al. (2001) apresentaram cinco classes de indicadores de desempenho: indicadores de avaliação de ordens de pedidos, indicadores de parcerias na cadeia de suprimentos, indicação e medição de níveis de produção, indicadores financeiros e indicadores de desempenho de entrega. Este último faz parte do estudo deste trabalho, por englobar alguns indicadores de desempenho logísticos. Além disso, podem fazer parte de indicadores de desempenho logístico: o tempo de ciclo 
de pedidos, dias de estoque, freqüência e prazo de entregas, entrega na data prometida, número de entregas completas e sem erro, custo de armazenagem e movimentação de mercadorias, entre outros (MENTZER; KONRAD, 1991; SIMATUPANG; SRIDHARAM, 2002; COYLE, BARD; LANGLEY, 2003; SIMATUPANG; SRIDHARAN, 2005).

Lee e Whang (2000) destacaram alguns indicadores logísticos como: desempenho das entregas (em tempo, completas e sem erro), tempo de ciclo, cobertura de estoque e cumprimento de agenda. Outras informações, como volume e previsão de vendas, também foram citadas como relevantes para a composição dos indicadores de desempenho.

Autores como Ellram (1990), Lambert (1993), Sauvage (2003), Barratt (2004), Simatupang e Sridharan (2005), Pigatto (2005) e Vieira (2006) avaliaram indicadores referentes à colaboração logística entre parceiros de uma cadeia de suprimento.

\section{MATERIAL E MÉTODOS}

Este trabalho constitui uma pesquisa quantitativa a respeito da colaboração logística entre uma distribuidora e seus fornecedores (indústrias de medicamentos e nãomedicamentos), por meio da aplicação de um questionário estruturado, aplicado aos fornecedores. Foi realizada uma revisão literária em artigos e teses que aborde indicadores de desempenho logístico e relacionados à parceria, bem como uma coleta de informações na distribuidora sobre variáveis de interesse para serem medidas. Com esse conjunto de variáveis, foi elaborado o questionário que servirá como pré-teste para validação do questionário final.

\subsection{Formulação de variáveis de colaboração logística}

Este estudo teve início durante a realização de um estágio supervisionado no local, por um período de um ano e cinco meses. Dessa forma, a coleta de informações ocorreu de forma bem peculiar e dentro das reais necessidades da distribuidora. Essas informações foram obtidas por meio de conversas informais com diretores, gerentes e colaboradores da empresa.

Os diretores enfatizaram a construção de um relacionamento com seus parceiros que tivessem o objetivo de atingir uma integração entre as organizações, destacando variáveis como confiança, reciprocidade e flexibilidade, já citadas em Vieira (2006), acrescentadas à variável comprometimento. Outra observação importante foi a freqüente comunicação entre as áreas financeiras (da Distribuidora e da indústria), pois se têm notado maior agilidade e entendimento entre as áreas comerciais da indústria e a de compras da distribuidora do que entre as áreas financeiras.

As variáveis relacionadas a transporte, como: envolvimento e estabilização das cargas em filme esticável (stretch), transporte de cargas na forma paletizada e uso de operador logístico comum, foram mencionadas pelos diretores como formas de facilitar e agilizar as descargas dos caminhões. Variáveis relacionadas a pedidos - possibilidade de trabalhar com pedidos pendentes e possibilidade de atender a pedidos homogêneos - foram destacadas em conversas com o gerente de compras. As demais variáveis foram inspiradas no trabalho de Vieira (2006).

\subsection{Projeto questionário}

O questionário foi aplicado a uma amostra de 34 fornecedores, dentro de um universo de 75 deles. A aplicação do questionário ocorreu por meio de telefone, e-mail ou diretamente na empresa. O projeto questionário é composto por três blocos, cada um deles com os objetivos descritos subseqüentemente.

Bloco 1: é composto por quatro questões (variáveis) e visa caracterizar a indústria da pesquisa e servir como variáveis de controle para futuras análises estatísticas. As variáveis deste bloco são:

Setor: os setores são divididos em medicamentos, que se subdividem em similares e genéricos, e nãomedicamentos, que se subdividem em cirúrgicos, homecare, acessórios, dermocosméticos, conveniência e oficinais.

Localização da empresa: esta variável influencia o desempenho logístico, uma vez que, à medida que aumenta a distância para entrega dos produtos, a complexidade de atender a um pedido perfeito aumenta.

Número de funcionários e faturamento anual: variáveis que denotam simetria, ou seja, quer dizer escala (tamanho e estrutura da organização) e posição no setor. Um alto grau de simetria entre as empresas se refere ao poder que cada uma exerce no mercado.

Bloco 2: este bloco tem por objetivo avaliar a variabilidade e a freqüência das respostas do questionário 
sobre o tema pesquisado, por meio de variáveis para controlar as percepções dos entrevistados, sendo composto por sete variáveis, descritas a seguir.

Volume de venda: esta variável analisa o desempenho logístico das indústrias, uma vez que um maior volume de venda resulta num maior volume e, geralmente, maior frequiência de entregas.

Q uantidade de produtos distribuídos: importante, pois, quanto maior o número de produtos distribuídos, maior a complexidade de cumprir as metas logísticas, porque aumenta a probabilidade de entregas incompletas, com erros e atrasadas.

F reqüência de pedidos: esta variável pode ser usada como medida de desempenho logístico por representar a eficiência no abastecimento por um período sem, necessariamente, aumentar o estoque, pois os produtos são entregues em menor quantidade, porém em um número maior de vezes.

Prazo de entrega: é uma variável que pode ser usada para medir o ciclo de pedido, ou seja, o intervalo entre a indústria receber o pedido e este ser entregue à distribuidora.

Reuniões logísticas: podem ser usadas para demonstrar que maior frequiência de reuniões aproxima os parceiros.

F reqüência de visitas técnicas: serve para revelar as potencialidades e oportunidades de novos negócios com o parceiro.

Terceirização: esta variável pode ser utilizada para esclarecer situações em que o fornecedor procura cumprir suas obrigações, entretanto a distribuidora recebe pedidos danificados ou atrasados.

Bloco 3: este bloco tem por objetivo avaliar a colaboração logística entre os parceiros, sob a ótica dos fornecedores.

Para captar os elementos de colaboração logística, algumas variáveis foram selecionadas por meio da revisão literária e outras durante o tempo de observação do relacionamento entre os parceiros (estágio supervisionado), formando quatro grupos e totalizando 28 variáveis. Cada grupo foi nomeado de forma intuitiva, com base no conjunto de variáveis que o compõe. Esses grupos estão relacionadas ao desempenho logístico operacional, à troca de informações logísticas e comerciais, à integração interpessoal e às variáveis referentes a pedidos. A seleção das variáveis por grupo foi feita por especialistas na área de Logística (professores, diretores e gerentes), sendo a maioria dos grupos análoga ao trabalho de Vieira (2006).

\subsection{Tratamento dos dados 3.3.1. Análise fatorial}

Segundo Pestana e Gageiro (2000), a análise fatorial é um instrumento que possibilita organizar a maneira como os sujeitos interpretam as coisas, indicando as que estão relacionadas entre si e as que não estão. Esta análise permite ver até que ponto diferentes variáveis têm subjacente o mesmo conceito (fator). A análise de componentes principais é indicada para casos em que se procura determinar um número mínimo de fatores que resulte no registro do maior possível de variância na análise (fatores esses que explicam a variação).

O modelo de análise fatorial (baseado em Pestana e Gageiro, 2000; Hair et al., 2005; e Johnson, 2002) pode ser escrito como:

$$
\begin{aligned}
& X_{1}=b_{11} F_{1}+b_{22} F_{2}+b_{1 k} F_{1 k}+U_{1} \\
& X_{2}=b_{21} F_{1}+b_{22} F_{2}+b_{2 k} F_{1 k}+U_{2} \\
& \dddot{X}=b_{p 1} F_{1}+b_{p 2} F_{2}+b_{p k} F_{1 k}+U_{p}
\end{aligned}
$$

em que $b_{i j}, i=1, \ldots, p ; j=1, \ldots, k$ representam a correlação entre os fatores comuns e as variáveis observáveis; $\mathrm{e}_{\mathrm{i}}, \mathrm{i}=1, \ldots, \mathrm{p}$ é o fator único. Os fatores únicos estão na forma padronizada, isto é, têm média zeroe variância unitária.

A análise fatorial decompõe a variância das variáveis observáveis em duas partes: uma relacionada aos fatores comuns e outra aos fatores únicos. A primeira conhecida como comunalidade de variável i, que é a variância total da variável explicada pelos fatores. E o outro é conhecido como complemento aritmético, que representa a proporção de variância única da variável, ou parte não explicada pelos fatores comuns.

Para extração dos fatores foi utilizada a análise dos componentes principais, que é indicada para casos em que se procura determinar um número mínimo de fatores que resulte no registro do maior número possível de variância na análise (fatores esses que explicam a variação) (HAIR, 2005). O primeiro fator contém o maior porcentual de explicação da variância total da amostra, sendo o segundo fator, por sua vez, o maior porcentual, e assim por diante. Assim, as variáveis são correlacionadas dentro de um fator comum e são praticamente independentes de outras que compõem outros fatores, de forma que a derivação destes contribui para maximizar a porcentagem de variância total relativa a cada fator consecutivo. Nesse processo, determinam-se as cargas fatoriais que relacionam as variáveis dentro de cada fator e têm a mesma função dos coeficientes de correlação. 
Para testar a adequação do modelo de análise fatorial, utiliza-se o teste de esfericidade de Barlett, em que, quanto mais próximo de zero for o nível de significância, maior será a adequação para o conjunto de dados, pois evidencia que existe correlação entre as variáveis. Outro teste de adequação da amostra é a medida de Kaiser-Meyer-Olkin (KMO), que compara as correlações simples e parciais observadas entre as variáveis.

De acordo com Pestana e Gageiro (2000), é possível criar índices que sumarizam a informação dada pelos itens que os integram. Esses índices são resultados de médias ponderadas pelas cargas fatoriais da matriz de componentes rotacionados. Para o fator j, pode-se criar um índice $\mathrm{lj}$, de acordo com a equação 1 .

$I_{j}=\frac{\sum_{n} b_{i j} X_{i}}{\sum_{i=1}^{n} b_{i j}} i=1,2, \ldots p . j=1,2, \ldots k \quad$ (equação 1) em que:

$\mathrm{p}=$ número de variáveis e $\mathrm{k}=$ número de fatores;

bij = carga fatorial da variável i e do fator $\mathrm{j}$;

$\mathrm{Xi}=$ variável que compõe $\mathrm{o}$ fator; $\mathrm{e}$

$\mathrm{I}=$ índice $\mathrm{j}$.

Para realizar as análises estatísticas, utilizou-se o software SPSS (Statistic Package for Social Study, versão 12.0) como ferramenta de análise fatorial dos dados do questionário.

\section{RESULTADO E DISCUSSÃO 4.1. A Distribuidora}

A distribuidora está localizada na cidade de Valença, RJ, no eixo Rio de Janeiro-São Paulo-Minas Gerais. Dessa forma, consegue um fluxo rápido de mercadorias e entrega dos pedidos, através das estradas e rodovias que a ligam à Região Sudeste. As farmácias e drogarias são as potenciais compradoras da empresa.
Atua em todo o Estado do Rio de Janeiro, em Minas Gerais (Zona da Mata) e São Paulo (Vale do Paraíba), atendendo os mais de 3.500 clientes cadastrados, sendo $78 \%$ no Estado do Rio de Janeiro. Este Estado constitui seu foco de distribuição onde, atualmente, tem grande poder de pulverização.

Os produtos distribuídos constituem medicamentos genéricos e similares e não medicamentos como oficinais, dermocosméticos, acessórios cirúrgicos, de conveniência e Homecare. Essas categorias de produtos totalizam 1.900 itens, dos quais $28 \%$ são medicamentos e $72 \%$, não-medicamentos.

Atualmente, a empresa representa e distribui para 75 fornecedores, sendo alguns deles com distribuição exclusiva no Estado do Rio de Janeiro. O setor de medicamento constitui $9,1 \%$ do total de fornecedores e corresponde a $40 \%$ do faturamento. No entanto, o setor de não-medicamento possui $90,1 \%$ dos fornecedores e corresponde a $60 \%$ do faturamento.

\subsection{Apresentação dos dados}

Os resultados são apresentados e discutidos em duas seções. Nesta primeira seção (4.2), faz-se uma apresentação dos dados referentes à caracterização da empresa (Bloco 1) e à caracterização do relacionamento entre a distribuidora e seus fornecedores (Bloco 2). $\mathrm{Na}$ segunda seção (4.3), faz-se uma análise das estatísticas básicas dos quatro agrupamentos baseados na revisão literária, relacionando-os com os dados dos Blocos 1 e 2 e uma análise desses agrupamentos por meio da análise fatorial.

De um total de aproximadamente 70 fornecedores, foram contatados 57 , dos quais 34 retornaram o questionário, correspondendo a $59,65 \%$, ou seja, uma taxa de resposta significativa. Os contatos ocorreram por telefone ou diretamente com os representantes das empresas. Já as respostas foram obtidas por entrevistas diretas ou por telefone e por meio de correio eletrônico (Tabela 1).

Tabela 1 - Obtenção dos dados

\begin{tabular}{|l|c|c|c|}
\hline \multicolumn{1}{|c|}{ Obtenção de dados } & Contato Dir eto & Contato por telefone & \% da forma de obtenção dos dados \\
\hline Entrevistas diretas & 4 & - & 11,76 \\
\hline Entrevistas por telefone & - & 1 & 2,94 \\
\hline Via correio eletrônico & 6 & 23 & 85,29 \\
\hline \% do meio de comunicação & $29,41 \%$ & 70,59 & 100,00 \\
\hline
\end{tabular}

Fonte: Elaborada pelos autores. 
Apesar de 85,29\% das entrevistas terem sido realizadas por meio de correio eletrônico, todas as empresas apresentam contato direto com a distribuidora, por meio de seus representantes de venda, e todas as questões foram explicadas aos participantes antes do preenchimento.

\subsubsection{Caracterização das indústrias}

O levantamento dos dados foi realizado com diretores, gerentes, supervisores e analistas das áreas de logística e comercial da indústria. Os profissionais da área comercial que participaram do levantamento mostram grande integração e contato com a área de logística das respectivas indústrias. Na Tabela 2, apresenta-se o número de cargos por setor de atuação da indústria.

Observou-se que o número de representantes de cargos mais estratégicos (diretores e gerentes) corresponde a 19 e se mostra superior ao dos cargos menos estratégicos (supervisores e analistas), que correspondem a 15.
Para dimensionar as indústrias, utilizaram-se, como critério, o número de funcionários e o faturamento anual, que foi colocado como uma questão opcional para os respondentes. Foram obtidos poucos dados de faturamento, por isso o indicador utilizado foi o número de funcionários. Na Tabela 3 , apresenta-se a porcentagem de empresas localizadas no Estado de São Paulo, Rio de Janeiro ou Minas Gerais e outros estados, assim com a porcentagem de empresas com maior ou menor dimensão.

Observou-se que $55,88 \%$ das indústrias estão localizadas no Estado de São Paulo, o que levou a distribuidora a ter um operador logístico, em Guarulhos, para otimizar as coletas de mercadorias. Notou-se que $52,94 \%$ das indústrias são do setor de não-medicamento e estão no Estado de São Paulo. Isso contribuiu para essa decisão estratégica, uma vez que as dimensões dos itens não-medicamentos se mostram muito maiores do que os dos medicamentos, o que gera um volume de carga por pedido enorme. A distribuidora tem maior número de parceiros de pequeno a médio porte, uma vez que $58,82 \%$ das empresas entrevistadas apresentaram um número de funcionários abaixo de 200.

Tabela 2 - Participação dos respondentes por setor de atuação

\begin{tabular}{|l|c|c|c|c|c|}
\hline Setor de atuação & Diretor & Gerente & Supervisor & A nalista & Total geral \\
\hline M edicamentos & 1 & 4 & 1 & - & 6 \\
\hline Não-medicamentos & 3 & 11 & 5 & 9 & 28 \\
\hline Total geral & 4 & 15 & 6 & 9 & 34 \\
\hline
\end{tabular}

Fonte: Elaborada pelos autores.

Tabela 3 - Dimensionamento das indústrias

\begin{tabular}{|c|c|c|c|c|}
\hline \multirow{2}{*}{ Setor de atuação } & \multicolumn{2}{|c|}{ L ocalização da indústria } & \multicolumn{2}{|c|}{ Número de funcionários } \\
\hline & Fora do Est. de São Paulo & Estado de São Paulo & Abaixo de 200 & Acima de 200 \\
\hline M edicamento & $14,71 \%$ & $2,94 \%$ & $11,76 \%$ & $5,88 \%$ \\
\hline Não-medicamento & $29,41 \%$ & $52,94 \%$ & $47,06 \%$ & $35,29 \%$ \\
\hline Total geral & $44,12 \%$ & $55,88 \%$ & $58,82 \%$ & $41,18 \%$ \\
\hline
\end{tabular}

Fonte: Elaborada pelos autores.

Observou-se que 55,88\% das indústrias estão localizadas no Estado de São Paulo, o que levou a distribuidora a ter um operador logístico, em Guarulhos, para otimizar as coletas de mercadorias. Notou-se que 52,94\% das indústrias são do setor de não-medicamento e estão no Estado de São Paulo. Isso contribuiu para essa decisão estratégica, uma vez que as dimensões dos itens não-medicamentos se mostram muito maiores do que os dos medicamentos, o que gera um volume de carga por pedido enorme. A distribuidora tem maior número de parceiros de pequeno a médio porte, uma vez que $58,82 \%$ das empresas entrevistadas apresentaram um número de funcionários abaixo de 200. 


\subsubsection{Caracterização do relacionamento indústria e distribuidora}

O ambiente de transação entre a distribuidora e os parceiros pode ser caracterizado pelas variáveis de quantidade e faturamento. Essas variáveis são o volume de venda que a distribuidora representa no faturamento total do parceiro, a porcentagem de produtos comercializados para a distribuidora em relação ao mix total da indústria e a freqüência de pedidos que a indústria recebe da distribuidora. Dessa forma, pode-se observar quanto a distribuidora representa no negócio da indústria. Conseqüentemente, quanto maior o volume transacionado, maior o envolvimento dos parceiros no negócio.

$\mathrm{O}$ volume de vendas, em porcentagem, que a distribuidora representa no montante da indústria, para cada setor de atuação, está dividido em faixas de valores menores ou maiores que $25 \%$, de acordo com a freqüência de volume de vendas. Na Figura 1, mostra-se o número de indústrias com um volume de venda maior ou menor que $25 \%$, em cada setor.

A quantidade de produtos vendidos, em porcentagem, para a distribuidora, em relação ao mix completo da indústria, foi segmentada em faixas de valores maiores ou menores que $25 \%$. Na Figura 2, mostra-se o número de indústrias com representação de mais ou menos $25 \%$ do seu mix, para cada setor de atuação da indústria.

$\mathrm{O}$ setor de medicamento, representado por seis indústrias, apresenta seu volume de venda dividido, ou seja, para metade dessas indústrias a distribuidora tem participação em seus negócios acima de $25 \%$ e para a outra metade, abaixo de $25 \%$. Nesse setor, a distribuidora comercializa mais de $25 \%$ do mix de todas as indústrias entrevistadas, ou seja, como elas represen$\operatorname{tam} 9,1 \%$ do universo de fornecedores da pesquisa e $40 \%$ do faturamento da distribuidora, sendo este um dado relevante, pois revela um comprometimento entre as partes que pode levar a uma eficiência no desempenho logístico. No setor de não-medicamento, maior parte das indústrias tem seus negócios representadas por menos de $25 \%$ do volume de vendas pela distribuidora, mesmo porque são muitas indústrias que representam esse setor. Notou-se que, quanto mais itens a distribuidora comercializar, em relação ao mix total da indústria, maior será sua participação no negócio da indústria.

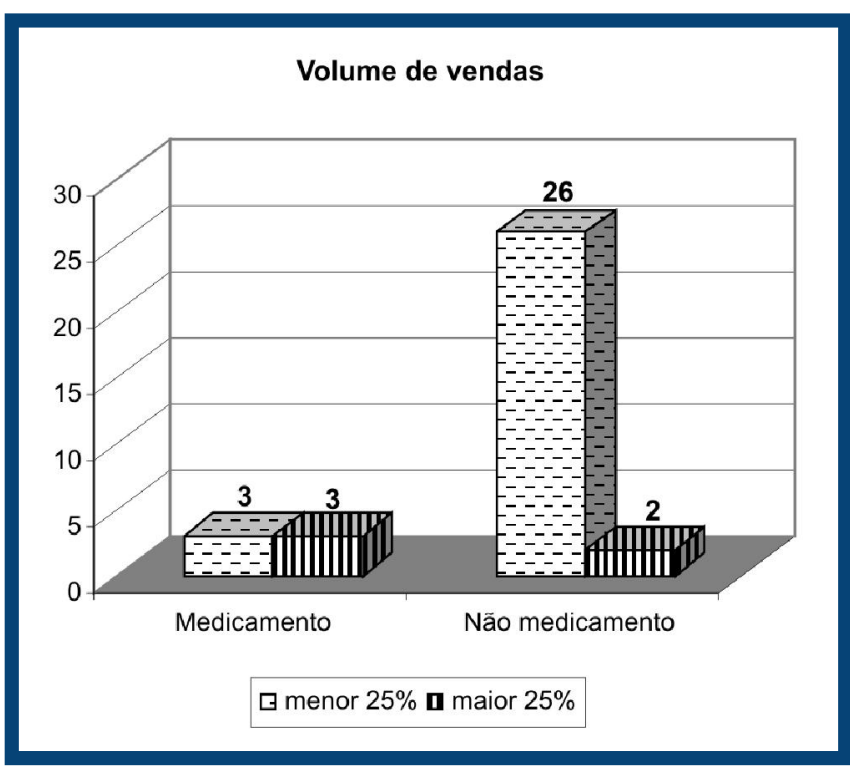

Figura 1 - Volume de vendas que o cliente distribuidor representa para a indústria.

Fonte: Elaborada pelos autores.

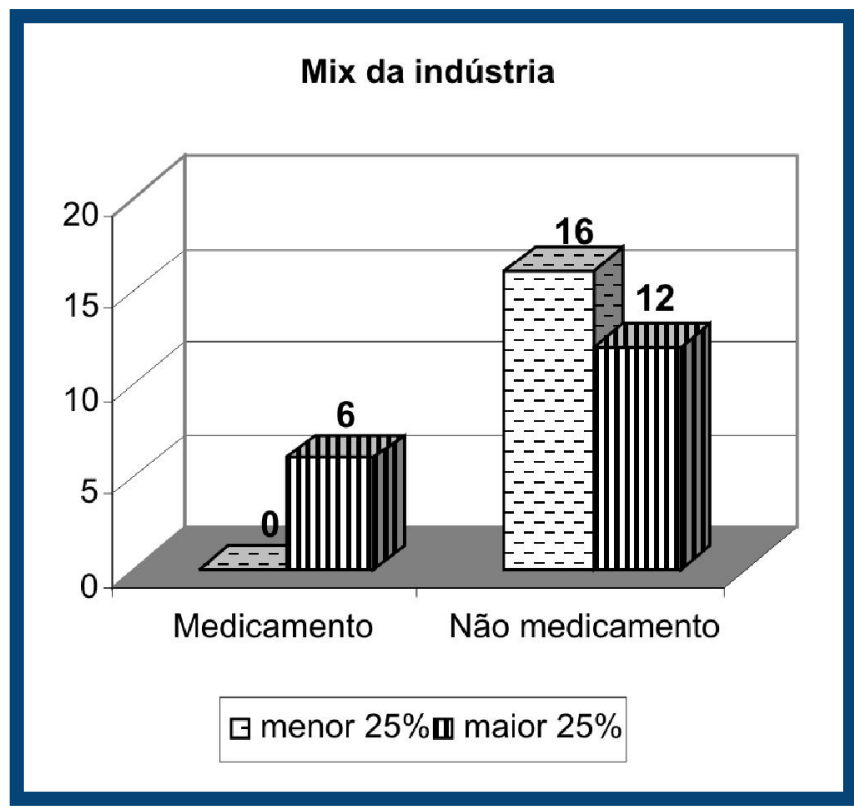

Figura 2 - Quantidade de itens vendidos pela Distribuidora em relação ao mix total da indústria.

Fonte: Elaborada pelos autores.

A frequiência de pedidos que a indústria recebe da distribuidora foi dividida em períodos maiores ou menores do que 15 dias, para cada setor de atuação. $\mathrm{O}$ setor de medicamento apresenta 50,0\% das indústrias com pedidos em um intervalo menor que 15 dias, e 50,0\% com pedidos em intervalo maior que 15 dias. Já o setor de não-medicamento possui $42,86 \%$ das indústrias com 
pedidos em um intervalo menor que 15 dias e $57,14 \%$ acima de 15 dias. Observou-se um ambiente com baixa transação de mercadoria, entre a indústria e a distribuidora, na metade dos fornecedores respondentes. Isso gera um baixo envolvimento entre os parceiros, seguido de uma escassa troca de informações logísticas.

O prazo de entrega dos produtos para a distribuidora pode variar em um período acima ou abaixo de cinco dias. Dessa forma, o setor de medicamentos possui $33,33 \%$ das suas indústrias com um prazo de entrega abaixo de cinco dias e $66,67 \%$ das indústrias com um prazo de entrega acima de cinco dias. O setor de não-medicamento apresenta $75,0 \%$ das indústrias com um prazo abaixo de cinco dias e $25 \%$ acima de cinco dias. Esses resultados indicaram que as indústrias de não-medicamento têm a capacidade de atender o seu cliente distribuidor mais rápido do que as de medicamento, apesar de gerar um volume de carga por pedido muito maior.

O grau de conhecimento do parceiro pode ser representado pela freqüência de reuniões entre os parceiros e pela regularidade de visitas realizadas entre eles. Na Tabela 4, explana-se quanto a indústria partilha de conhecimento com a distribuidora.

Observa-se, nessa tabela, que há uma baixa ocorrência de reuniões logísticas entre os parceiros, distribuidora e indústrias, tanto no setor de não-medicamento quanto no de medicamento. Isso influência diretamente o desempenho logístico, por causa da dificuldade de conhecer as estratégias dos parceiros, a malha logística, as formas de armazenagem e até as estratégias desenvolvidas entre as indústrias e outros parceiros distribuidores, bem como dificulta também a troca de informações e contribui para a menor relação de interdependência e flexibilidade. Em se tratando dos fornecedores de não-medicamentos, que somam $60 \%$ do faturamento da distribuidora e possuem menor prazo de entrega, maior contato com a distribuidora é fundamental para melhorar a sua eficiência logística.

\subsection{Análise dos grupos}

Como descrito na metodologia, os agrupamentos foram baseados na literatura e no caso peculiar de parceria entre a distribuidora e seus fornecedores. Na Tabela 5, mostram-se a média, o desviopadrão, a mediana e o terceiro quartil das variáveis correspondentes a cada grupo. Esses valores indicam a percepção de cada respondente em relação à parceria.

Na Tabela 5, mostram-se os melhores valores do Grupo 2 (elementos de integração interpessoal) e Grupo 4 (elementos referentes a pedidos). Em relação ao Grupo 2, segundo os entrevistados, há uma satisfação na parceria quanto ao relacionamento entre as partes, mesmo que se tenham baixa frequiência de reuniões logísticas e pouca ocorrência de visitas técnicas. Quanto ao Grupo 4, os entrevistados também disseram que os pedidos da distribuidora são sempre atendidos. No entanto, a execução desses pedidos parece não atender, de forma satisfatória, os entrevistados, como evidenciam os valores do Grupo 1 (elementos operacionais de desempenho logístico), pois estes apresentam baixa média e alta variabilidade dos dados, em comparação com os demais grupos. Ao analisar individualmente as variáveis dos Grupos 1 e 2, observou-se que esses fornecedores são, em sua maioria pequenas empresas, o que pode revelar baixo investimento em operações com paletização e uso de filme, baixa utilização de caixas ou contenedores (facilitadores de transporte), baixo uso de sistemas de informação etc. Esses grupos tiveram as suas variáveis analisadas por meio de fatores. Logo, a técnica de análise fatorial foi empregada com os objetivos de reduzir o número de variáveis desses grupos, coincidentemente os de maior número de variáveis, e, assim, facilitar a compreensão do fenômeno, tendo em vista que a realidade das análises subseqüentes se torna mais fácil quando se trabalha com poucos fatores.

Tabela 4 - Dados de conhecimento da indústria com a distribuidora

\begin{tabular}{|l|c|c|c|c|}
\hline \multirow{2}{*}{ Setor de atuação } & \multicolumn{2}{|c|}{ Ocorrência de reunião logística } & \multicolumn{2}{|c|}{ Intervalo de visitas } \\
\cline { 2 - 5 } & Já teve & Nunca teve & Mensal & Não-mensal \\
\hline Medicamento & $11,76 \%$ & $5,88 \%$ & $11,76 \%$ & $5,88 \%$ \\
\hline Não-medicamentos & $11,76 \%$ & $70,59 \%$ & $38,24 \%$ & $44,12 \%$ \\
\hline Total geral & $23,53 \%$ & $76,47 \%$ & $50,00 \%$ & $50,00 \%$ \\
\hline
\end{tabular}

Fonte: Elaborada pelos autores. 
Tabela 5 - Avaliação dos atributos de colaboração logística por meio de medidas centrais

\begin{tabular}{|c|c|c|c|c|c|}
\hline Código & Variável de colaboração logística & M édia & Desvio-padrão & M ediana & 3Q uartil \\
\hline & Grupo 1 - Elementos oper. de desempenho logístico & & & & \\
\hline DL1 & Entrega na data prometida & 8,17 & 1,38 & 8,00 & 9,25 \\
\hline DL2 & Entrega completa & 8,67 & 1,24 & 9,00 & 10,00 \\
\hline DL3 & Entregas sem erro & 8,94 & 1,04 & 9,00 & 10,00 \\
\hline DL4 & Tempo de entrega & 8,32 & 1,45 & 8,00 & 10,00 \\
\hline DL5 & Operador logístico comum & 5,52 & 4,23 & 8,00 & 9,00 \\
\hline DL6 & Facilitadores de transporte & 6,76 & 3,23 & 8,00 & 9,00 \\
\hline DL7 & Tempo carregamento & 8,14 & 1,90 & 8,50 & 9,00 \\
\hline DL8 & Carga na forma paletizada & 5,76 & 3,21 & 6,00 & 9,00 \\
\hline \multirow[t]{2}{*}{ DL9 } & Filme esticável (strech) & 5,55 & 3,21 & 5,50 & 8,25 \\
\hline & Grupo 2 - Elementos de integração interpessoal & & & & \\
\hline DL10 & Comprometimento & 8,91 & 1,99 & 10,00 & 10,00 \\
\hline DL11 & Confiança & 9,50 & 0,99 & 10,00 & 10,00 \\
\hline DL12 & Reciprocidade & 9,20 & 1,20 & 10,00 & 10,00 \\
\hline \multirow[t]{2}{*}{ DL13 } & Flexibilidade & 8,97 & 1,33 & 9,00 & 10,00 \\
\hline & Grupo 3 - Elementos de inf. logísticas e comerciais & & & & \\
\hline DL14 & Troca de informações logísticas & 6,26 & 2,81 & 7,00 & 8,25 \\
\hline DL15 & Compartilhamento das inf. de níveis de estoque & 6,23 & 2,83 & 7,00 & 8,00 \\
\hline DL16 & Participação de equipes nos processos logísticos & 5,64 & 3,32 & 7,00 & 8,00 \\
\hline DL17 & Transparência na comunicação & 8,73 & 1,46 & 9,00 & 10,00 \\
\hline DL18 & Compartilhamento das inf. na troca de previsão de vendas & 7,32 & 2,44 & 8,00 & 9,00 \\
\hline DL19 & Participação conjunta em seminários e congressos & 5,20 & 2,99 & 6,50 & 8,00 \\
\hline DL20 & E da alta gerência nos projetos logísticos & 7,02 & 2,80 & 8,00 & 9,00 \\
\hline DL21 & Compartilhamento das informações de armazenagem & 6,73 & 2,83 & 7,00 & 9,00 \\
\hline \multirow[t]{2}{*}{ DL22 } & Comunicação entre as áreas financeiras & 7,44 & 2,37 & 8,00 & 9,25 \\
\hline & Grupo 4 - Elementos referentes a pedidos & & & & \\
\hline DL23 & Pedidos urgentes & 8,85 & 1,55 & 9,00 & 10,00 \\
\hline DL24 & Disponibilidade de produto & 8,50 & 1,37 & 9,00 & 10,00 \\
\hline DL25 & Produtos avariados & 8,70 & 2,00 & 9,00 & 10,00 \\
\hline DL26 & Padrões de qualidade & 9,52 & 0,96 & 10,00 & 10,00 \\
\hline DL27 & Pedidos pendentes & 9,05 & 1,39 & 10,00 & 10,00 \\
\hline DL28 & Pedidos homogêneos & 9,02 & 1,24 & 9,50 & 10,00 \\
\hline
\end{tabular}

Os principais pressupostos para a aplicação da análise fatorial foram atendidos. Os testes de adequação de amostra (teste de esfericidade Barlett) e a medida de Kaise-Meyer-Olkin (KMO) evidenciaram que a análise fatorial é recomendável pelos valores expressados, como exposto na Tabela 6.

Segundo Pestana e Gageiro (2000), valores com nível de significância de 0,000 a 0,05 levam a rejeição da hipótese de a matriz das correlações na população ser a identidade, mostrando que existe correlação entre as variáveis, o que não acontece nesse caso. O Kaise-MeyerOlkim (KMO), que varia entre 0 e 1 , compara as correlações simples com as parciais observadas entre as variáveis. Os valores de KMO maiores que 0,5 são considerados aceitáveis.
Para melhorar a leitura e interpretação dos dados na forma de fatores, foi feita uma rotação Varimax, mantendo a ortogonalidade entre eles. A rotação não modifica a contribuição conjunta da variância dos fatores, apenas permite que eles se relacionem, mais claramente, com determinados grupos de variáveis, facilitando a análise da solução encontrada. As Tabelas 7 e 9 apresentam, respectivamente, os fatores dos grupos (G1 e G3) e as cargas fatoriais, que correlacionam as variáveis com os fatores antes da rotação. Os valores das comunalidades, proporção da variância de cada variável explicada pelas componentes principais, sendo igual à soma dos quadrados das cargas fatoriais, são expressos em outra coluna. Na última linha, encontra-se a proporção da variância total explicada para cada fator. 
Na Tabela 7, apresentam-se os três fatores (F1, F2 e F3) com cargas acima de 0,5, consideradas estatisticamente satisfatórias. As variáveis que compõem o fator F1 são as mesmas discutidas na Revisão. Já as que compõem os fatores F2 e F3 são algumas peculiares ao caso da distribuidora, com exceção da variável tempo de carregamento (DL27). As comunalidades das variáveis de cada subgrupo (fator) indica, no geral, que os fatores são capazes de explicar grande parte da variância de cada série de dados, e a porcentagem da variância acumulada $(67,04 \%)$ indica que os três fatores são capazes de diferenciar os elementos operacionais de desempenho logístico em três subgrupos.

O primeiro fator (F1) explica $25,41 \%$ da variabilidade total e correlaciona-se, positivamente, com: entrega na data prometida (DL1), entrega sem erro (DL3), tempo de entrega (DL4) e entrega completa (DL2). Esse fator pode ser interpretado como uma medida do grau de interferência das entregas no desempenho logístico. Entretanto, esse fator está correlacionado, negativamente, com as variáveis facilitadores de entrega (DL6) e cargas pal etizadas (DL8). Esse resultado indica as empresas que possuem maiores desempenhos nas entregas e não têm interferência dos facilitadores de transporte e da paletização das cargas, mas possuem maiores escores fatoriais.
Conforme apresentado na metodologia, na equação 1 o primeiro indicador foi calculado a partir dos valores das cargas fatoriais de F1. Esse indicador foi denominado Indicador de Entrega - IE, pois está diretamente relacionado às variáveis que expressam as formas e tempos das entregas dos fornecedores.

O segundo fator (F2) explica 22,02\% da variância total e associa-se com: facilitadores de transporte (DL6), operador logístico comum (DL5) e tempo de carregamento (DL7). Em resumo, esse fator explica uma medida do grau de interferência do transporte no desempenho logístico. De forma análoga ao IE, realizouse o cálculo do segundo indicador derivado de F2, relacionado aos aspectos de transporte e denominado Indicador de Transporte - IT.

O terceiro fator (F3) explica 19,61\% da variabilidade total e está correlacionado com: filme esticável (DL9) e cargas paletizadas (DL8). Ou seja, esse fator explica uma medida do grau de interferência da forma de transportar cargas com o desempenho logístico. Esse fator também deu origem a umindicador, derivado de suas cargas fatoriais e denominado Indicador de Forma de Transportar Cargas - IFTC.

$\mathrm{Na}$ Tabela 8, apresentam-se as estatísticas descritivas dos três indicadores calculados a partir dos fatores extraídos da Tabela 7.

Tabela 6 - Medida de KMO e teste de esfericidade Barlett

\begin{tabular}{|l|c|c|}
\hline \multicolumn{1}{|c|}{ Grupos } & KM O & Barlett \\
\hline Grupo1 - Elementos operacionais de desempenho logístico & 0,543 & 0,000 \\
\hline Grupo3 - Elementos de informações logísticas e comerciais & 0,781 & 0,000 \\
\hline
\end{tabular}

Fonte: Elaborada pelos autores.

Tabela 7 - Resultado da análise fatorial do Grupo 1 (G1)

\begin{tabular}{|c|l|c|c|c|c|}
\hline \multicolumn{2}{|c|}{ G1 - Elementos oper acionais de desempenho logístico } \\
\hline \multirow{2}{*}{ VAR } & \multicolumn{1}{|c|}{ Variáveis } & \multicolumn{3}{c|}{ Cargas Fatoriais } \\
\cline { 2 - 6 } & & F1 (G1) & F2 (G1) & F3 (G1) & Comunalidade \\
\hline DL1 & Entrega na data prometida & 0,831 & 0,093 & $-0,092$ & 0,707 \\
\hline DL3 & Entrega sem erro & 0,722 & 0,083 & 0,146 & 0,550 \\
\hline DL4 & Tempo de entrega & 0,704 & $-0,155$ & $-0,124$ & 0,536 \\
\hline DL2 & Entrega completa & 0,540 & 0,285 & $-0,345$ & 0,492 \\
\hline DL6 & Facilitadores de transporte & $-0,223$ & 0,895 & 0,077 & 0,856 \\
\hline DL5 & Operador logístico comum & 0,114 & 0,756 & 0,105 & 0,596 \\
\hline DL7 & Tempo de carregamento & 0,409 & 0,636 & 0,179 & 0,603 \\
\hline DL9 & Filme esticável & 0,083 & 0,070 & 0,950 & 0,914 \\
\hline DL8 & Cargas na forma paletizada & $-0,224$ & 0,282 & 0,806 & 0,779 \\
\hline & Porcentagem de variância & $25,41 \%$ & $22,02 \%$ & $19,61 \%$ & \\
\hline
\end{tabular}

Fonte: Elaborada pelos autores. 
Tabela 8 - Estatística descritiva dos indicadores

\begin{tabular}{|l|c|c|}
\hline \multicolumn{1}{|c|}{ Indicadores } & Média & Desvio-padrão \\
\hline Indicador de Entrega (IE) & 8,60 & 1,14 \\
\hline Indicador de Transporte (IT) & 6,83 & 2,20 \\
\hline Indicador de Forma de Transportar Cargas (IFTC) & 5,30 & 3,31 \\
\hline
\end{tabular}

Fonte: Elaborada pelos autores.

Para verificar se as médias são estatisticamente diferentes, utilizou-se o teste t emparelhado. $\mathrm{O}$ teste de Kolmogorov-Sminorv para normalidade indicou que os indicadores são normais a 1\% de significância estatística. Embora a escala não tenha distribuição normal, a soma das variáveis tende a uma distribuição normal (Lei dos Grandes Números; MOOD; GRAYBILL, 1974). Logo, a análise do comportamento da normalidade pode ser feito pela Teorema do Limite Central (para $n>30$ ).

$\mathrm{O}$ teste $\mathrm{t}$ emparelhado foi significativo a $1 \%$ nas comparações das médias dos indicadores aos pares. A hipótese nula é: "a média de dois indicadores são iguais". Como Ho foi rejeitada, isso significa dizer que o valor de IE é superior ao IT e ao IFTC, o que permite afirmar que os aspectos relacionados à entrega apresentam maior grau de interferência no desempenho logístico dos fornecedores do que os aspectos de transporte e de forma de transportar as cargas. Por sua vez, o IT é superior, em média, ao IFTC, o que contribui para maior investigação quanto à forma de transportar as cargas. A baixa média referente aos IFTC pode ser explicada pelo fato de não existir reuniões logísticas entre 76,47\% dos parceiros e, principalmente, pela baixa ocorrência de visitas técnicas, ou seja, o maior conhecimento da parte operacional poderia minimizar os erros no momento de transportar os produtos. Na Tabela 9, mostram-se os dois fatores extraídos do Grupo 3, com cargas fatoriais acima de 0,5 e 65,08\% de variância acumulada.

As baixas cargas fatoriais das variáveis: troca de informações logísticas (DL14) e participação de equipes nos processos logísticos (DL16) acontecem pelo fato de estas estarem dividindo carga entre os dois fatores. Essa divisão de cargas fatoriais não apresenta justificativa plausível dentro do cenário avaliado. Talvez se o tamanho da amostra fosse maior, essas variáveis (DL14 e DL16) se correlacionariam mais com um dos fatores (F1 ou F2). $\mathrm{O}$ fator $\mathrm{F} 1$ está relacionado mais ao contato direto por meio de reuniões logísticas e o fator $\mathrm{F} 2$, às visitas técnicas. Ambos, o baixo índice de reuniões logísticas e a pouca ocorrência de visitas técnicas, certamente contribuíram para a divisão de cargas. Essas variáveis correlacionadas com os respectivos fatores $\mathrm{F} 1$ e F2 indicam um potencial para colaboração em termos de informações logísticas e comerciais, ou seja, a partir do contato direto entre as partes e da promoção de ações conjuntas podem elevar o grau de parceria, aumentar a integração interpessoal e, consequientemente, contribuir para maior eficiência dos elementos operacionais de desempenho logístico.

Tabela 9 - Resultado da análise fatorial do Grupo 3 (G3)

G3 - Elementos de infor mações logísticas e comer ciais

\begin{tabular}{|c|l|c|c|c|}
\hline \multirow{2}{*}{ VAR } & & \multicolumn{2}{|c|}{ Cargas fatoriais } & \multirow{2}{*}{ Comunalidade } \\
\cline { 3 - 5 } DL18 & Compartilhameis & F1 (G3) & F2 (G3) & Comto das informações de previsão de vendas \\
\hline DL22 & Comunicação entre as áreas financeiras & 0,841 & 0,166 & 0,735 \\
\hline DL15 & Compartilhamento das informações de níveis de estoque & 0,776 & 0,166 & 0,629 \\
\hline DL17 & Transparência na comunicação & 0,755 & 0,319 & 0,672 \\
\hline DL14 & Troca de informações logísticas & 0,660 & 0,118 & 0,449 \\
\hline DL20 & Envolvimento da diretoria nos projetos logísticos & 0,626 & 0,426 & 0,574 \\
\hline DL21 & Compartilhamento das informações de armazenagem & $-0,031$ & 0,884 & 0,783 \\
\hline DL19 & Participação conjunta em seminários e congresso & 0,374 & 0,775 & 0,740 \\
\hline DL16 & Participação de equipes nos processos logísticos & 0,382 & 0,714 & \\
\hline & & 0,539 & 0,573 & 0,655 \\
\hline
\end{tabular}

Fonte: Elaborada pelos autores. 
De forma análoga aos fatores do Grupo 1, foram obtidos indicadores relacionados ao Grupo 3 e realizado $o$ teste $\mathrm{t}$ de amostra emparelhada para verificar se as médias são iguais. O resultado foi significativo a $1 \%$ e evidenciou que o Indicador de Reuniões L ogísticas - IRL, derivado do fator F1 (G3), apresenta maior grau de interferência na troca de informações logísticas e comerciais do que do Indicador de Visita Técnica IVT, derivado do fator F2 (G3). Ou seja, a realização de reuniões logísticas pode contribuir, significativamente, para uma melhoria na troca de tais informações.

Tendo em vista que aproximadamente $90 \%$ dos fornecedores são pequenas indústrias, do ramo de nãomedicamentos, responsáveis por $60 \%$ do faturamento, possuem um prazo de entrega menor que o das demais empresas, estão localizadas, na sua maioria, no Estado de São Paulo e, em geral, os produtos possuem carga unitizada de alto volume, a utilização desses indicadores pela distribuidora para avaliar o desempenho logístico desses fornecedores poderia ser útil e trazer ganhos de competitividade diante de outras distribuidoras do ramo.

\section{CO NCLUSÃO}

A eficiência logística ganha cada vez mais importância nesse cenário, em que a competição não acontece somente entre uma empresa e outra, mas também entre um grupo de empresas que buscam um melhor nível de serviço para o seu cliente (distribuidora), que por sua vez também procura atender os seus clientes (drogarias, farmácias etc.) nas condições (tempo, quantidades e forma) desejadas. Em fase de crescimento e inserida nesse mercado, a distribuidora vem ampliando sua quantidade de fornecedores e, daí, a necessidade de parâmetros para avaliar o desempenho logístico desses fornecedores aumenta.

Este estudo mostrou ser possível medir o desempenho logístico dos parceiros da distribuidora, por meio de indicadores. A pesquisa aplicada apontou indicadores que mensuram o grau de interferência das entregas (IE), de transporte (IT), da forma de transporte de cargas (IFTC) (todos esses relacionados ao desempenho operacional logístico) e indicadores que podem mensurar o grau de interferência na troca de informações logísticas e comerciais (indicador de reuniões logísticas - IRL e indicador de visita técnica - IVT).

No geral, os resultados da análise fatorial, referentes aos grupos de desempenho logístico e troca de informações logísticas e comerciais, indicaram uma dire- ção para a implementação de políticas que possam melhorar o processo de entrega dos fornecedores. Isso evidencia que questões como entregas na data prometida, entregas sem erro, entregas completas e o tempo de entrega, ações conjuntas entre os parceiros e constante troca de informações operacionais merecem atenção especial no planejamento logístico da distribuidora. Investimentos nessa área são essenciais, criando e monitorando parâmetros que possam melhorar o desempenho logístico dos fornecedores.

Além de comparar e monitorar o desempenho logístico entre os fornecedores, os indicadores servem para aproximar os parceiros, ampliando a integração interpessoal e intensificando as trocas de informações logísticas.

O relacionamento entre a distribuidora e seus parceiros se mostrou mais forte entre as áreas comerciais do que entre as áreas logísticas. Isso pode ser notado pela ocorrência de reuniões logísticas com apenas 23,53\% dos fornecedores. $\mathrm{O}$ ambiente de transação entre a distribuidora e os seus parceiros foi caracterizado por uma baixa participação da distribuidora nos negócios de seus parceiros, de modo geral. No entanto, a distribuidora comercializa mais de $25 \%$ do mix total de 18 dos seus fornecedores, que corresponde a 53\% dos entrevistados.

O estabelecimento de variáveis ainda não pesquisadas e peculiares ao caso da distribuidora constitui uma contribuição teórica deste trabalho. A determinação de indicadores de desempenho possibilita que a distribuidora tenha critérios práticos de avaliação de seus parceiros, o que evidencia maior atenção de seus gestores na realização de novos negócios e na recontratação dos serviços já existentes.

A pesquisa quantitativa foi realizada, do ponto de vista dos fornecedores, como uma auto-avaliação. Isso pode gerar algumas informações contrárias dentro do ponto de vista da distribuidora em relação às variáveis avaliadas. O baixo número de entrevistas (34 respondentes) constitui uma restrição estatística do estudo, o que dificulta o detalhamento dos resultados por setor e por volume de vendas, entre outros parâmetros que poderiam ser analisados como variáveis de controle, no caso de amostra maior.

Com relação a trabalhos futuros, podem-se realizar outras técnicas de análise, como a de agrupamentos; estender este trabalho a outras cadeias de suprimentos; avaliar a colaboração logística entre os pares, por meio de estudo de caso; e aprofundar a pesquisa para os grupos que apresentaram menor média e maior variabilidade dos dados, por meio da aplicação de questionários aos demais fornecedores que não participaram da pesquisa. 


\section{REFERÊNCIAS}

ANDERSON, J. C.; NARUS, J. A. A model of distributor firm and manufacturer firm working partnerships. J ournal of $M$ arketing, v.54, p.42-58, 1990.

ÂNGELO, L. B. Indicadores de desempenho logístico. Florianópolis: GELOG-UFSC, 2005. Dissertação (Mestrado) Universidade Federal de Santa Catarina, Florianópolis.

BALLOU, R. H. Logística empresarial: transporte, gerenciamento de materiais e distribuição física. Trad. por Elias Pereira. 4. ed. Porto Alegre: Bookman, 2001. 532 p.

BASTOS, I. D. Avaliação do desempenho logístico do serviço de transporte rodoviário de cargas - Um estudo de caso no setor de revestimentos cerâmicos. Florianópolis: UFSC, 2003. 168f. Dissertação (Mestrado) - Universidade Federal de Santa Catarina, Florianópolis.

BARRATT, M.; OLIVEIRA, A. Exploring the experiences of collaborative planning initiatives. International J ournal of Physical Distribution \& Logistics M anagement, v. 31, number 4, p. 266-89, 2001.

BARRATT, M. Understanding the meaning of collaboration in the supply chain Supply C hain M anagement: an international journal, v. 9, n. 1 , p. 30-42, 2004

BOWERSOX, D. J. L ogística empresarial: o processo de integração da cadeia de suprimentos. São Paulo: Atlas, 2001.

BOWERSOX, D. J.; CLOSS, D. J.; STANK, T. P. How to master cross-enterprise collaboration. Supply C hain M anagement R eview, v. 1, n, 4, p.18-30, jul./aug. 2003.

COYLE, J. J.; BARD, E. J.; LANGLEY, C. J. JR. The management of business logistics: a supply chain perspective. $7^{\text {th }}$. Ohio: Thompson Learning, 2003. 707 p.

DAUGHERTY, P. J.; ELLINGER, A. E.; GUSTIN, C. M. Integrated logistics: achieving logistics performance improvements. Supply Chain M anagement, v. 1, n. 3, p. 25-33, 1996.

ELLRAM, L. M. International supply chain management: strategic implications for the purchasing function, Dissertation presented in partial fulfillment of the requirements for the degree of Doctor of Philosophy in the Graduate School of the Ohio State University. New York: Ohiostate University. [s.d.]. $343 \mathrm{f}$.

FUNDAÇÃO PARA O PRÊMIO NACIONAL DA QUALIDADE (FPNQ). Indicadores de desempenho. São Paulo: FPNQ, 1995.

GANESAN, S. Determinants of long-term orientation in buyer-seller relationships. J ournal of M arketing, v. 58, n. 2, p.1-19, april, 1994.

GUNASEKARAN, A.; PATELL, C.; TIRTIROGLU, E. Performance measures and metrics in a supply chain environment. International J ournal of Operations \& productions M anagement, v. 21, n. 1/2, p. 71-87, 2001.

HAIR, J.; ANDERSON, R.; TAHTAM, R.; BLACK, W. A nálise multivariada de dados. Tradução por Adonai S. Sant'Anna e Anselmo C. Neto. 5. ed. Porto Alegre: Bookman, 2005. 593 p.
HEIDE, J. B.; JOHN, G. Do norms matter in marketing relationships? J ournal of M arketing, v. 56, n. 2, p. 32-44, april, 1992.

HRONEC, S. M. Sinais vitais. São Paulo: Makron Books, 1997.

JOHNSON, R.A.; WICHERN, D.W. Applied multivariate statistical analysis. New Jersey: Prentice Hall, 2002.

JURAN, J. M. Planejamento para a qualidade. São Paulo: Pioneira, 1992.

KUMAR, N. The power of trust in manufacturer-retailer relationship. Harvard Business Review, p. 92-106, nov./dec. 1996.

LAMBERT, D. M. Developing a customer-focused logistics strategy. International J ournal of Physical Distribution and Logistics M anagement, v. 23, n. 6, p. 12-19, 1993.

LEE, H. L.; WHANG, S. Information sharing in a supply chain. International J ournal of Technology M anagement, v. 20, n. 3, p. 373-387, 2000.

MACEDO-SOARES, T. D. A.; RATTON, C. Medição de desempenho e estratégias orientadas para o cliente. ER A-R evista deA dministração de Empresas, São Paulo, v. 39, n. 4, p. 46-59, out./dez. 1999.

MENTZER, J. T.; KONRAD, B. P. An efficiency/effectiveness approach to logistics performance analysis. J ournal of Business Logistics, v. 12, n. 1, p. 33-62, 1991.

MENTZER, J. T.; FOGGIN, J. H.; GOLICIC, S. L. Collaboration: the enablers, impediments, and benefits. Supply $C$ hain $M$ anagement Review, v. 4, n. 4, p. 52-57, 2000.

MOHR, J.; SPEKMAN, R. Characteristics of partnership success: partnership attributes, communication behavior, and conflict resolution techniques. Strategic M anagement J ournal, v. 15, n. 2, p. 135-152, 1994.

MORGAN, R. M.; HUNT, S. D. The commitment - trust theory of relationship marketing. J ournal of $M$ arketing, v. 58, n. 3, p. 2038, jul. 1994.

NOVAES, A. G. Logística e gerenciamento da cadeia de distribuição: estratégia, operação e avaliação. Rio de Janeiro: Campus, 2001. 409 p.

OHMAE, K. The global logic of strategic alliances. Harvard Business Review, p.143-155, mar./apr. 1989.

PESTANA, Maria H.; GAGEIRO, João N. Análise de dados para ciências sociais: a complementaridade do SPSS. 2. ed. Lisboa, 2000. p.143-155.

PIGATTO, G. Avaliação de relacionamentos no canal de distribuição de produtos de mercearia básica. São Carlos, SP: UFSCar, 2005. 239 f. Tese (Doutorado em Engenharia de Produção) Universidade Federal de São Carlos, São Carlos.

REY, M. Indicadores de desempenho logístico. São Paulo: Movimentação \& Armazenagem, 1999. p. 86-90. 
SAUVAGE, T. The relationship between technology and logistics third-party providers. International J ournal of Physical Distribution \& Logistics M anagement, v. 33, n. 3, p. 236-253, 2003.

SIMATUPANG, T. M.; SRIDHARAN, R. The collaborative supply chain. International J ournal of L ogistics M anagement, v. 3, n. 1, p. 15-30, 2002.

SIMATUPANG, T. M.; SRIDHARAN, R. The collaboration index: a measure for supply chain collaboration. International J ournal of Physical Distribution \& L ogistics M anagement, v. 35, n. 1, p. 44-62, 2005.

SPEKMAN, T. E.; KAMAUFF JR., J. W.; MYHR, N. An empirical investigation into supply chain management. International J ournal of Physical Distribution and Logistics M anagement, v. 28, p. 630-650, 1998.
VIEIRA, J. G. V. Avaliação do estado de colaboração logística entre indústrias de bens de consumo e redes de varejo alimentar. São Paulo: USP, 2006. Tese (Doutorado) - Universidade São Paulo, São Paulo.

VIEIRA, J. G. V.; YOSHIZAKI, H. T. Y.; HO, L. L. Avaliação do estudo de colaboração logística entre a indústria de bens de consumo e rede de varejo supermercadista. BT/PRO/0611. Boletim Técnico da USP, 2007. 207 p.

ZANQUETTO, H. F.; FEARNE, A.; PIZZOLATO, N. D. The measurement of benefits from and enablers for supply chain partnerships in the UK fresh produce industry. Chain and Network Science, p. 59-74, 2003. 